\title{
Patches in the polar ionosphere: UT and seasonal dependence
}

\author{
J. J. Sojka, M. D. Bowline, and R. W. Schunk \\ Center for Atmospheric and Space Sciences, Utah State University, Logan
}

\begin{abstract}
The seasonal and UT dependencies of patches in the polar ionosphere are simulated using the Utah State University time dependent ionospheric model (TDIM). Patch formation is achieved by changing the plasma convection pattern in response to temporal changes in the interplanetary magnetic field (IMF) $B_{y}$ component during periods of southward IMF. This mechanism redirects the plasma flow from the dayside high-density region, which is the source of the tongue of ionization (TOI) density feature, through the throat and leads to patches, rather than a continuous TOI. The model predicts that the patches are absent at winter solstice (northern hemisphere) between 0800 and 1200 UT and that they have their largest seasonal intensity at winter solstice between 2000 and 2400 UT. Between winter solstice and equinox, patches are strong and present all day. Patches are present in summer as well, although their intensity is only tens of percent above the background density. These winter-to-equinox findings are also shown to be consistent with observations. The model was also used to predict times at which patch observations could be performed to determine the contributions from other patch mechanisms. This observational window is \pm 20 days about winter solstice between 0800 and 1200 UT in the northern hemisphere. In this observational window the TOI is either absent or reduced to a very low density. Hence the time dependent electric field mechanism considered in this study does not produce patches, and if they are observed, then they must be due to some other mechanism.
\end{abstract}

\section{Introduction}

Buchau et al. [1983] have shown that polar $F$ region density structures come in two main forms, patch and sun-aligned arc structures. The patches were found to correlate with times of strong geomagnetic activity. Today, we also know that the patches form under southward IMF conditions. Weber et al. [1984] showed that the patches were transported into the polar cap, rather than being formed locally by precipitation. Foster and Doupnik [1984] observed plasma transport into the cusp region, through it, and into the polar cap. This transport of plasma, specifically patches from the cusp into the polar cap, was also observed by Weber et al. [1986]. Patches were observed to have a strong diurnal modulation when viewed from Thule in the center of the polar cap at winter solstice [Buchau et al., 1985].

These observations show that the patches convect across the polar cap at the prevailing convection speed. This convection is predominantly antisunward for southward interplanetary magnetic field (IMF) conditions. Such convection can be traced backward, through the cusp, into a dayside region equatorward of the auroral ionosphere. Hence the origin of the patches could be equatorward of the cusp region, within the cusp region, or a combination of both. Weber et al. [1984, 1986] had already shown that patches do not originate inside the polar cap. Indeed, Tsunoda [1988] synthesized a descriptive model of large-scale patches by including a combination of mechanisms. First, plasma transport convects high-density, solar-produced plasma from lower latitudes into the cusp. Historically, the feature that develops in association with this transport through the cusp

\footnotetext{
Copyright 1994 by the American Geophysical Union.

Paper number 93JA03327.

0148-0227/94/93JA-03327\$05.00
}

and across the polar cap is referred to as the "tongue of ionization" (TOI). Sato [1959] identified this region of enhanced polar cap plasma in maps of ground-based $f_{0} F_{2}$ and proposed that the "tongue" was caused by solar-illuminated plasma drifting into the polar cap. Knudsen [1974] showed how magnetospheric convection and cusp precipitation combine to produce the observed TOI densities. This solarproduced phenomenon has a strong UT modulation, and hence in different longitude sectors its intensity is different. This was modeled by Sojka and Schunk [1982], observed by de la Beaujardiere et al. [1985], and is consistent with the patch UT modulation observed by Buchau et al. [1985]. More recently, Foster [1993] has shown that in addition to this diurnal effect, the midlatitude afternoon sector from which the TOI plasma is derived is very sensitive to geomagnetic activity, specifically, storms. Foster showed that during storms the source region density is enhanced and demonstrated that these stormenhanced densities (SED) are transported to the cusp and, consequently, would form enhanced TOI. The location of the plasma source region was also found to move to lower latitudes as geomagnetic activity increases, hence changing the transport path to the cusp. Tsunoda's [1988] model also included cusp processes, with the result that the TOI could be made into patches. The mechanisms he considered were (a) soft electron precipitation, leading to enhanced plasma density; (b) enhanced electric fields, causing ion heating, enhanced plasma recombination, and hence density reduction; (c) changing IMF $B_{z}$ and $B_{y}$ components, which can relocate the cusp equatorward-poleward and east-west, respectively. It should be noted that this latter relocation of the cusp, by a changing IMF $B_{y}$ component, is the principal mechanism used in this study. Recently, A. S. Rodger et al. (manuscript in preparation, 1993) have presented observations showing the presence of the cusp precipitation mechanism, and Valladares et al. [1994] have shown the presence of density decreases associated with enhanced electric fields. 
Other authors have argued that electric field dynamics play the major role in the production of patches. Anderson et al. [1988] modeled how the sudden expansion of the convection electric field pattern could enable high-density plasma located equatorward of the cusp to be brought into the polar cap as a patch. This expansion of the convection pattern was associated with increases in the geomagnetic activity. During times of strong storm and substorm activity the midlatitude plasma density is modulated. Such enhanced or reduced plasma density regions have been observed moving toward the cusp from Millstone Hill [Foster, 1993]. This mechanism modulates the plasma that forms the TOI before it passes through the cusp. Sojka et al. [1993] modeled patch formation by allowing the convection pattern to change with changes in the IMF $B_{y}$ component. In so doing, the TOI became transformed into a sequence of patches, since the location at which the TOI entered the polar cap was a strong function of the convection pattern. These results from two independent model studies were presented by Decker et al. [1992], Sojka et al. [1992], and Bowline et al. [1992]. Lockwood and Carlson [1992] proposed that the patches are produced by transient magnetopause reconnection. This type of mechanism has the appeal of matching reconnection time constants with ionospheric patch scale sizes. Indeed, the flow channel events (FCE) used as a working scenario by A. S. Rodger et al. (manuscript in preparation, 1993) and Valladares et al. [1994] could be viewed as an ionospheric signature associated with reconnection.

Observationally and theoretically, the polar cap patch phenomenon is a tantalizing "weather" phenomenon. In the applications community it is also a noticeable bad weather effect. The steep density gradients found at the edges of the patches lead to instabilities and the generation of plasma irregularities at various smaller scale sizes. It is these irregularities which lead to severe problems in any application in which radio waves traverse the ionosphere. Finally, it should be noted that most of the present-day research dealing with polar cap patches stems from the National Science Foundation Coupling Energetics and Dynamics of Atmospheric Regions (CEDAR) High-Latitude Plasma Structure workshop held at Peaceful Valley, Colorado, in May 1992. At this meeting, almost all of the mechanisms discussed in this introduction were presented and discussed.

This study extends the work of Sojka et al. [1993] by modeling the UT and seasonal dependencies of polar cap patches. In this investigation the patches are formed by allowing an IMF $B_{y}$ variation to change the plasma convection pattern, thereby redirecting the TOI. Since the convection pattern changes faster (every $30 \mathrm{~min}$ ) than it takes for the TOI to set up (several hours), patches of ionization are produced. The modeled seasonal and UT variations are compared with those observed by Buchau et al. [1985]. The model is also used to predict when observations could be carried out to assess the contribution from other patch formation mechanisms.

\section{Time Dependent Ionospheric Model (TDIM)}

The ionospheric model was initially developed as a midlatitude, multi-ion $\left(\mathrm{NO}^{+}, \mathrm{O}_{2}^{+}, \mathrm{N}_{2}^{+}\right.$, and $\left.\mathrm{O}^{+}\right)$model by Schunk and Walker [1973]. The time dependent ion continuity and momentum equations were solved as a function of altitude for a corotating plasma flux tube including diurnal variations and all relevant $E$ and $F$ region processes. This model was extended to include high-latitude effects due to convection electric fields and particle precipitation by Schunk et al. [1975, 1976]. A simplified ion energy equation was also added, which was based on the assumption that local heating and cooling processes dominate (valid below $500 \mathrm{~km}$ ). Flux tubes of plasma were followed as they moved in response to convection electric fields. A further extension of the model to include the minor ions $\mathrm{N}^{+}$and $\mathrm{He}^{+}$, an updated photochemical scheme, and the mass spectrometer incoherent scatter (MSIS) atmospheric model is described by Schunk and Raitt [1980].

The addition of plasma convection and particle precipitation models is described by Sojka et al. [1981a, b]. Schunk and Sojka [1982] extended the ionospheric model to include ion thermal conduction and diffusion thermal heat flow. Also, the electron energy equation was included by Schunk et al. [1986], and consequently, the electron temperature is now rigorously calculated at all altitudes. The theoretical development of the TDIM is described by Schunk [1988], while comparisons with observations are discussed by Sojka [1989].

In addition to the convection input, the TDIM requires several other inputs. The auroral oval parameters, specifically the electron energy flux and average energy, were obtained from the Hardy et al. [1987] model. The geomagnetic activity was set at a $K_{p}$ of 3.5 . The MSIS-86 model was used to represent the neutral atmosphere [Hedin, 1987]. A solar maximum condition was assumed, represented by an $F_{10.7}$ index of 210. These additional inputs were all kept constant during the 24-hour TDIM simulations.

Each snapshot of the patch simulation region consists of 2035 pixels (bins) with each bin having an $\sim 70 \times 70 \mathrm{~km}$ size at an altitude of $100 \mathrm{~km}$. A single trajectory was followed for each pixel. Hence a total of 2035 trajectories were followed for over 24 hours to produce a single snapshot.

\section{Patch Formation}

This study of the seasonal and UT dependencies of polar cap $F$ region patches is a direct follow-up to the Sojka et al. [1993] patch formation study, which showed that patches could be formed by modulating the IMF $B_{y}$ component. The result of this convection modulation is to redirect the plasma flow associated with the polar cap tongue of ionization (TOI) as it enters the polar cap. Figure 1 shows such a TOI, as simulated by the TDIM, in the winter northern hemisphere at 1900 UT. In this case the convection model is the Heppner and Maynard [1987] unvarying "A" pattern. At this UT the plasma flux tubes convecting through the cusp into the darkness of the winter polar cap have previously been in sunlight at lower latitudes. This transports high-density plasma into the polar cap, which then convects antisunward for the IMF southward condition, to form the TOI. The peak electron density $\left(N_{m} F_{2}\right)$ in the TOI exceed $10^{6} \mathrm{~cm}^{-3}$ near the magnetic pole, while outside this region, the density falls to about $10^{5} \mathrm{~cm}^{-3}$.

If a different convection pattern, representing a change in the $B_{y}$ component, had been used, such as the Heppner and Maynard [1987] "DE" or "BC" patterns, the location of the TOI in the polar cap would be different. However, the plasma in the TOI takes several hours to convect across the polar cap, while the changes in the IMF $B_{v}$ component are typically much faster. Hence as new TOI orientations are formed at the cusp, the old TOI already in the polar cap drifts antisunward from its original orientation. This leads to the patch formation mechanism discussed by Sojka et al. [1993]. 

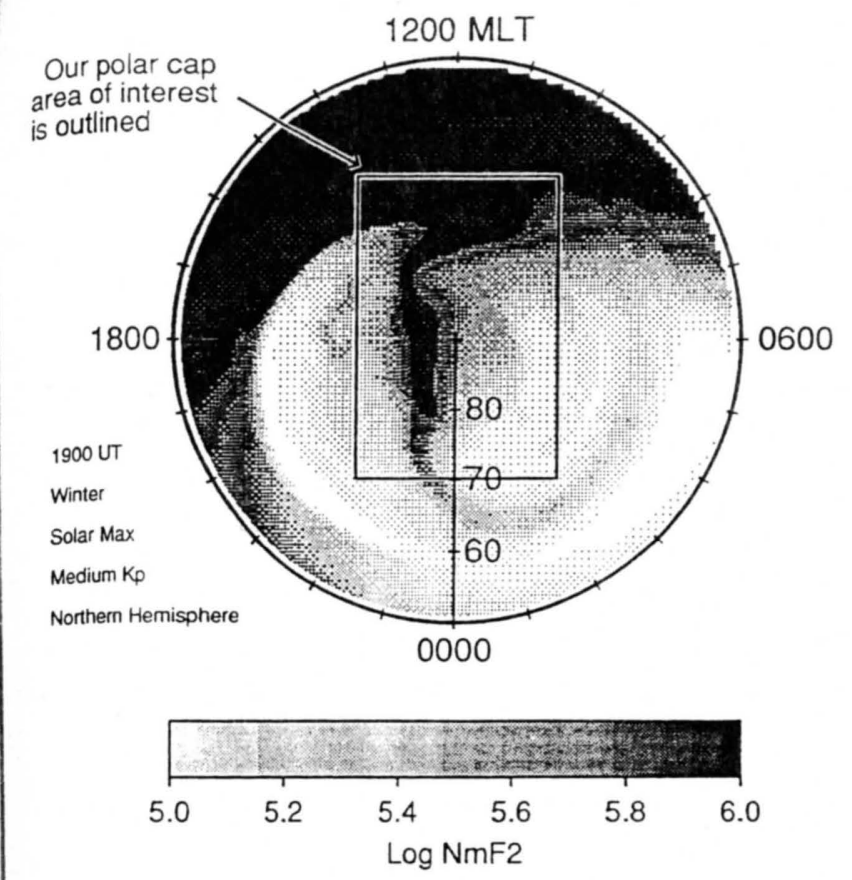

Figure 1. Snapshot of peak electron density $\left(N_{m} F_{2}\right)$ showing a tongue of ionization for the UT and seasonal conditions noted on the left-hand side. $N_{m} F_{2}$ is gray scaled according to the gray scale key. Subsequent figures and plates emphasize the rectangular polar cap region outlined here, which is referred to as the patch simulation region.

In this study we have adopted two specific convection patterns and changed from one to the other at half-hour intervals. The choice of a half-hour interval is based crudely on time constant arguments dictated by observations as follows. Time constants associated with dayside reconnection (perhaps flux transfer events, FTE) range from 8 to $20 \mathrm{~min}$. During this time period the cusp reconfigures and redirects $F$ region plasma transport. Given that the polar cap plasma speeds are of the order of a few hundred meters per second, then plasma features associated with these cusp changes should have lengths of a few hundred kilometers. For this study the convection was changed every half hour, with the expectation that the associated polar cap plasma features would have scale sizes approaching $1000 \mathrm{~km}$. The convection patterns are changed instantaneously; this was done owing to the lack of a more justifiable scheme. At this time no theoretical, largescale convection model has either the required temporal or spatial resolution. The assimilative mapping of ionospheric electrodynamics (AMIE) work of Knipp et al. [1993] has the potential of achieving this resolution but is still starved of observation data with adequate spatial coverage. Observations of the plasma convection changes near the cusp indicate rapid dynamics, with a timescale of tens of seconds to $\sim 1 \mathrm{~min}$. If these timescales are reasonable, then the phenomenon we are looking at, namely the large-scale transport of plasma through the cusp, will not be seriously affected by our instantaneous switching, since it is the longer timescales (tens of minutes) that are important in generating new TOI features. Most certainly, the rapidly varying "transitions" will generate additional fine structuring that we are not modeling. These would correspond to some of the other types of patch formation mechanisms described in the introduction. Patches can also be generated by varying the convection smoothly from one pattern to another. Although such patches tend to be smoother and less discrete, they are recognizable as equivalent to those presented here.

The two convection patterns adopted are the Heppner and Maynard [1987] A and DE patterns in the northern hemisphere, corresponding to southward IMF, with IMF $B_{y}$ being weakly and strongly negative, respectively. Figure $2 \mathrm{a}$, for the $\mathrm{A}$ pattern, shows the large-scale convection pattern as equipotentials, and Figure $2 \mathrm{~b}$ shows the plasma transport with corotation in the patch simulation region. The patch simulation region is defined in Figure 1. The length of each flow trajectory in Figure $2 \mathrm{~b}$ corresponds to $20 \mathrm{~min}$ of UT. The uniform antisunward flow across the polar cap is evident. Around noon the convection is predominantly westward at the lowest latitudes (Figure 2b, top), then it rotates and flows strongly eastward and poleward through the cleft-cusp region. Flow speeds of $\sim 1 \mathrm{~km} / \mathrm{s}$ in the cleft and $0.5 \mathrm{~km} / \mathrm{s}$ are found in the polar cap. Figure $3 \mathrm{a}$ shows the equivalent convection and Figure $3 \mathrm{~b}$ the plasma transport for the DE convection pattern. In this case of strong IMF $B_{y}$ negative the plasma speed reaches $2 \mathrm{~km} / \mathrm{s}$ in the dusk polar cap and drops to $200 \mathrm{~m} / \mathrm{s}$ in the dawn polar cap. The cleft flow speeds are also intensified, reaching a maximum speed of $\sim 3 \mathrm{~km} / \mathrm{s}$. Although the speed of the plasma flow changes from the A to the DE patterns, the direction of the flow is generally very similar. Clearly, had the $\mathrm{BC}$ (IMF $B_{y}$ positive) pattern been compared with the DE pattern, a dramatic change in the flow pattern would have been seen. However, using only the change from A to DE patterns and vice versa was shown by Sojka et al. [1993] to be more than adequate for the production of polar cap patches.

\section{UT and Seasonal Dependencies}

The TDIM was run for 24 hours for day 357 (December 23), with the convection pattern changing between A and DE every 30 min (Plate 1). In Plate 1, color-coded snapshots of simulated $N_{m} F_{2}$ are shown for 12 UTs. Each panel corresponds to the patch simulation region outlined in Figures 1,2 , and 3 . The UT is specified at the top of each panel. $N_{m} F_{2}$ is presented as a color plot of its logarithm to the base 10, as shown in the color key. The densities range from $\sim 10^{5}$ to $2 \times 10^{6} \mathrm{~cm}^{-3}$.

Because of the time varying convection, the smooth TOI shown in Figure 1 becomes highly structured; see the snapshot at 2000 UT in Plate 1 . Plate 1 demonstrates a very strong UT dependence. Around 0800 UT the densities are low $\left(\leq 3 \times 10^{5}\right.$ $\mathrm{cm}^{-3}$ ) and relatively unstructured within the polar cap. In contrast, around 1800 UT the densities range from $2 \times 10^{5}$ to 2 $\mathrm{x} 10^{6} \mathrm{~cm}^{-3}$, and large patches are present within the polar cap. Our earlier patch formation study [Sojka et al., 1993] concentrated on this latter time period. By inspection of Plate 1 the "strongest" patches are present between 1600 and 2400 UT, while the patches disappear between 0600 and 1000 UT. These times also correspond to those at which the TOI is at its maximum and minimum, respectively. The patches or density structures range in spatial extent from 1 to about 5 pixels in width (dawn-to-dusk) and from 10 to 30 pixels in length (noon-to-midnight). The patches are irregular (each pixel is $70 \mathrm{~km} \times 70 \mathrm{~km}$ ), hence the patch dimensions range from hundreds to $\sim 2000 \mathrm{~km}$ in scale size. These dimensions are consistent with those observed.

Plate 2 is a similar $N_{m} F_{2}$ simulation snapshot series, but this time is for equinox, day 82, (March 23). The $N_{m} F_{2}$ 

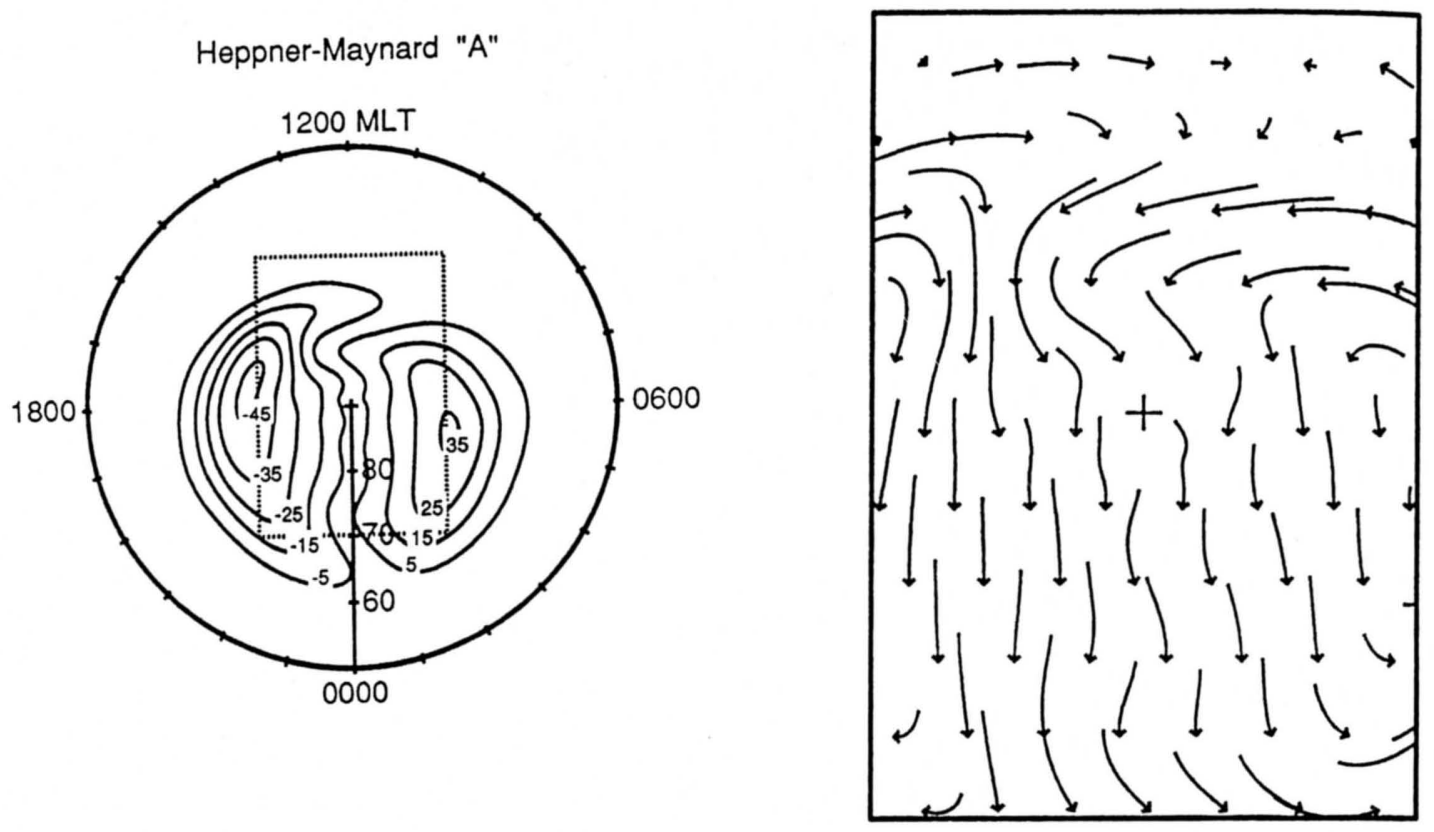

(a)

(b)

Figure 2. (a) The equipotential contours associated with the A convection pattern of Heppner and Maynard [1987]. (b) Detail of the corresponding plasma flow (including corotation) in the patch simulation region. Each flow arrow corresponds to $20 \mathrm{~min}$ of plasma transport.
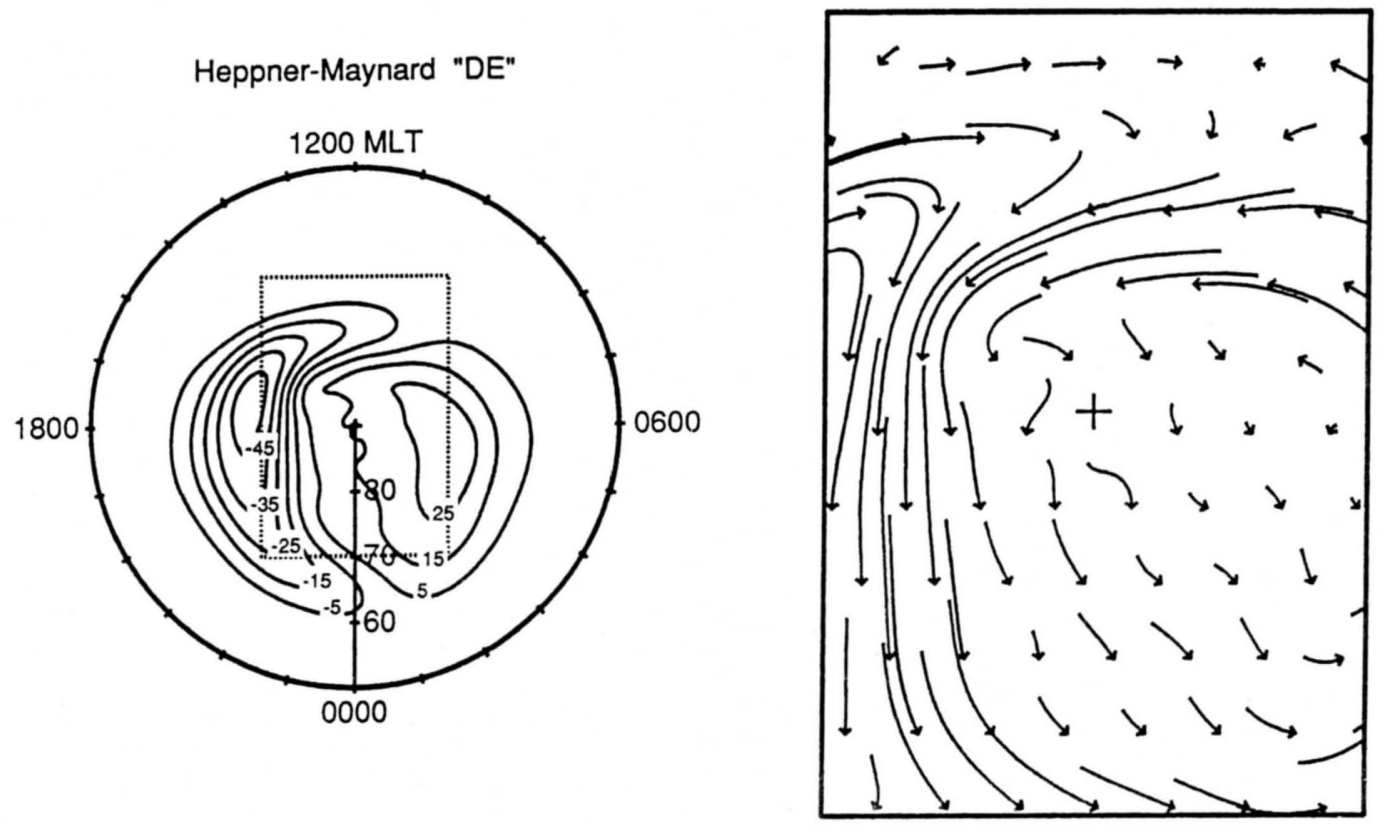

(a)

(b)

Figure 3. (a) Equipotential contours associated with the DE convection pattern of Heppner and Maynard [1987]. (b) Detail of the corresponding plasma flow (including corotation) in the patch simulation region. Each flow arrow corresponds to 20 min of plasma transport. 


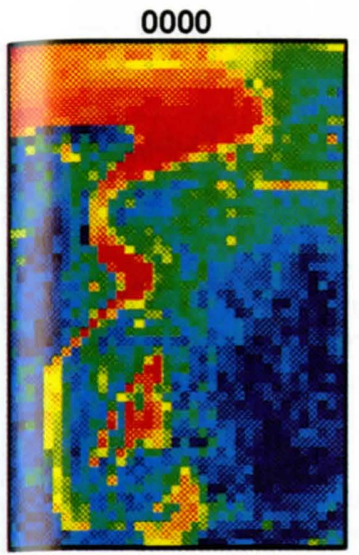

\section{0}

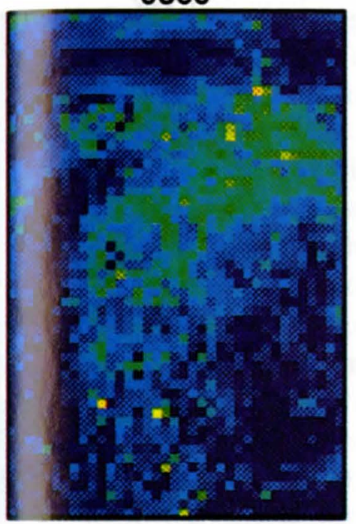

1600

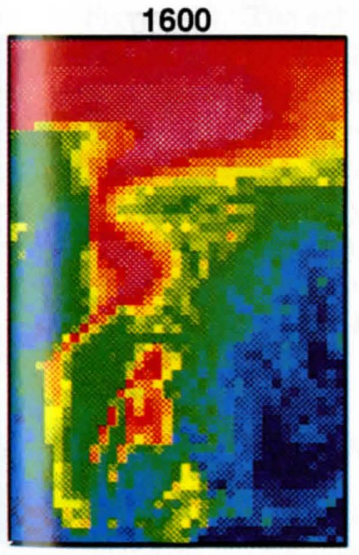

0200

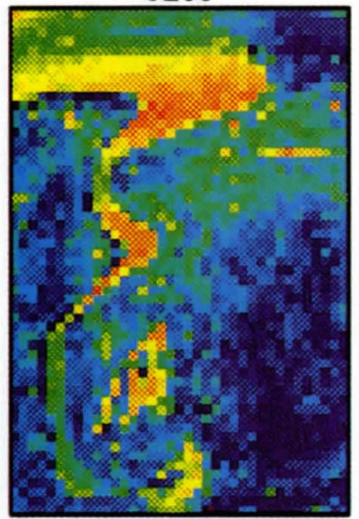

1000

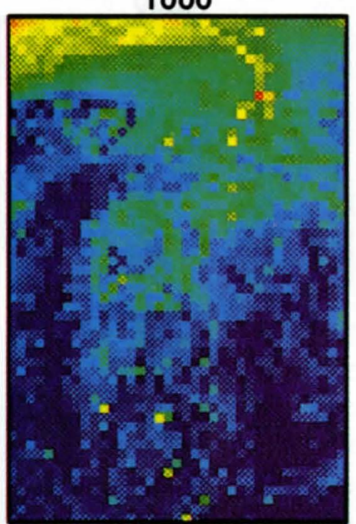

1800

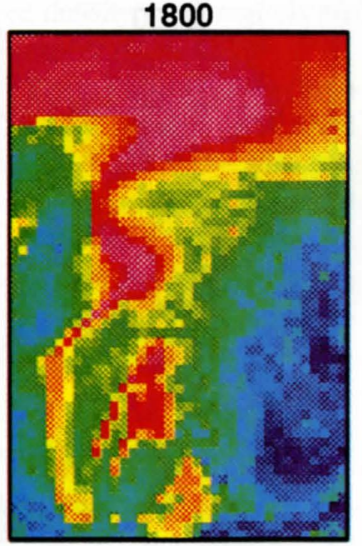

0400

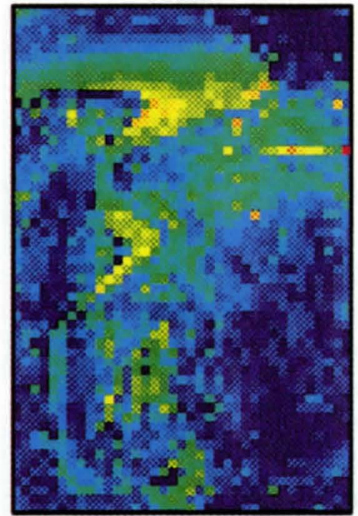

1200

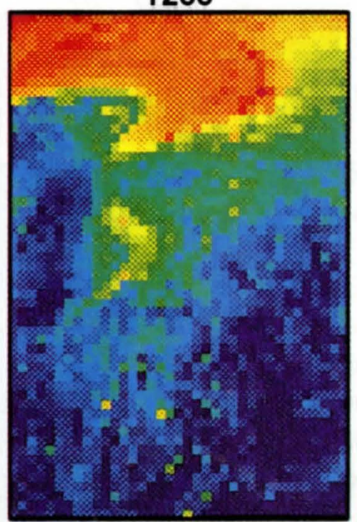

2000

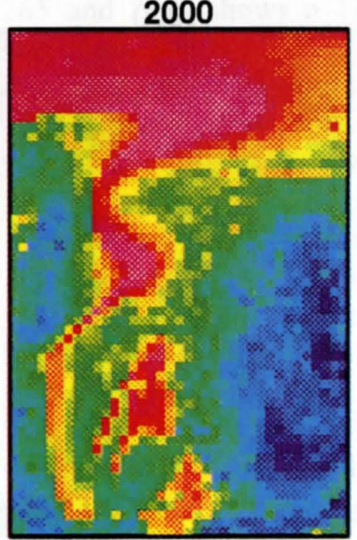

0600

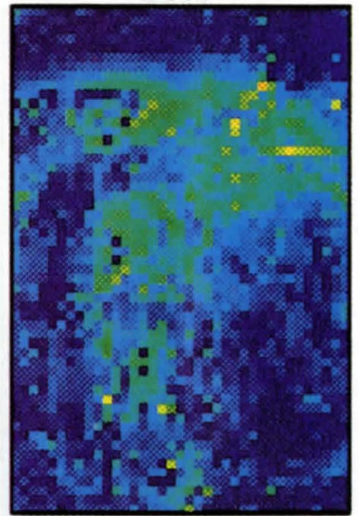

1400

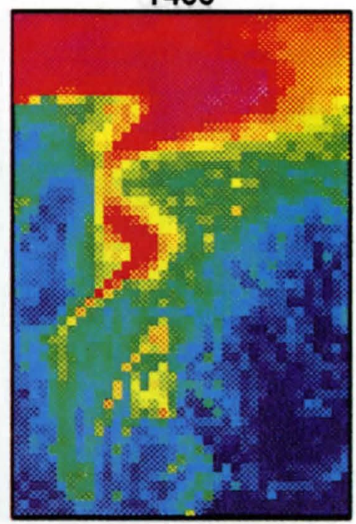

2200

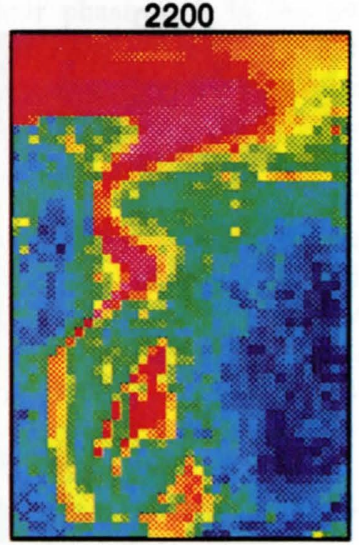

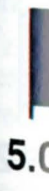

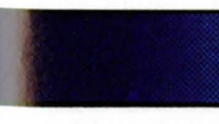

5.0

5.2

5.4

5.6

5.8

6.0

6.2

$\log _{10} \mathrm{NmF2}$

Plate 1. Peak electron density $\left(N_{m} F_{2}\right)$ in the patch simulation region over a 24-hour period for winter solstice and solar maximum conditions. The 12 panels are snapshots at 2-hour intervals. The patches were generated by changing the convection between the A and DE patterns of Heppner and Maynard [1987]. $N_{m} F_{2}$ is color coded and is presented in the coordinate system shown in Figure 1. 


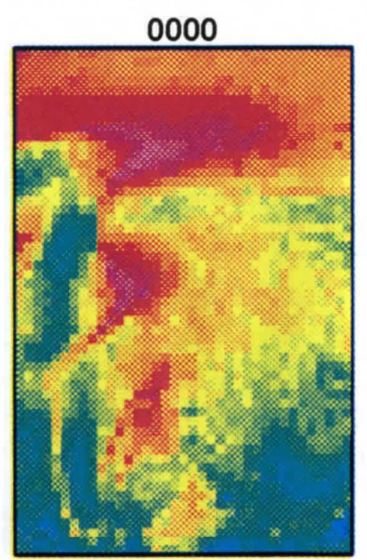

0800

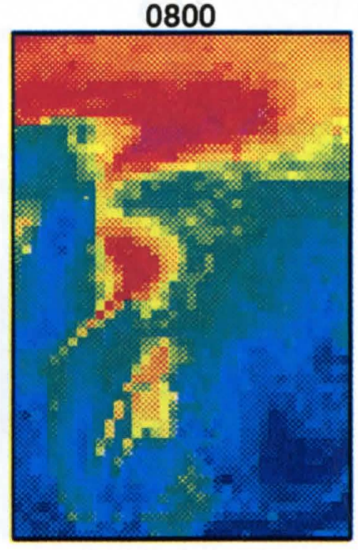

1600

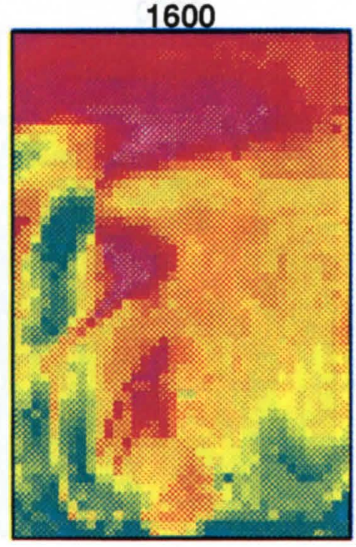

0200

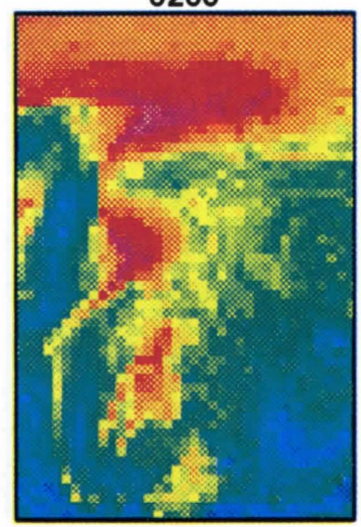

1000

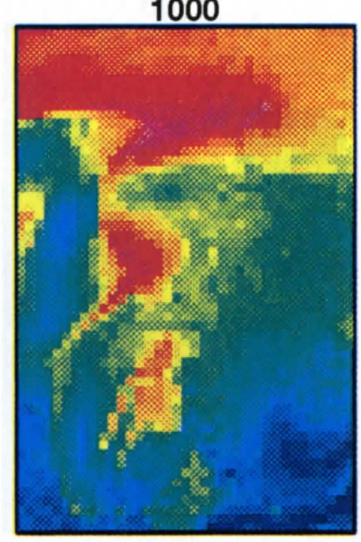

1800

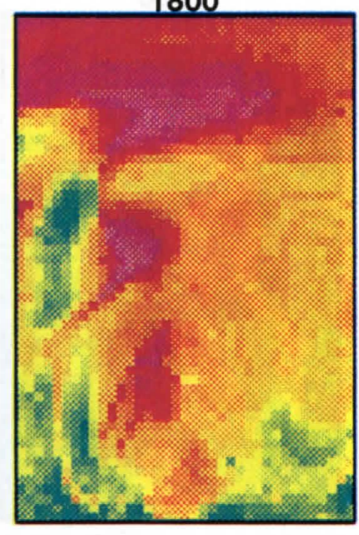

0400

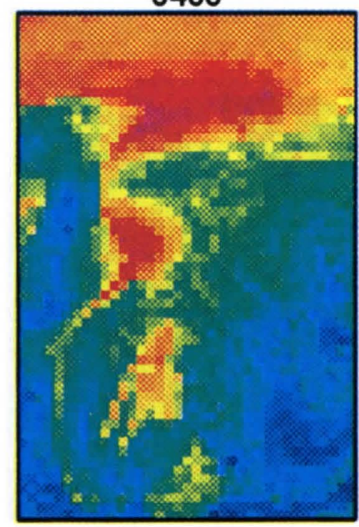

1200

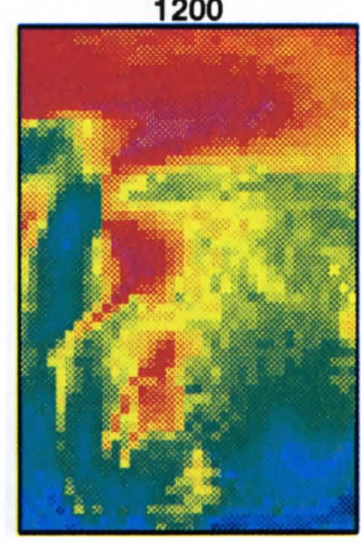

2000

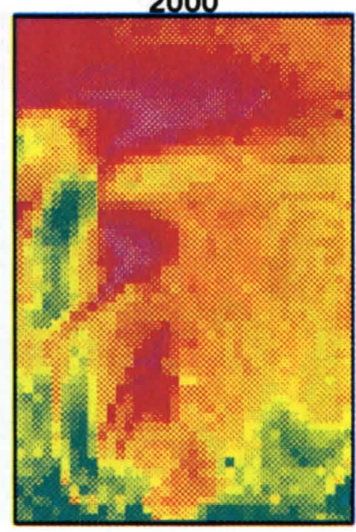

0600

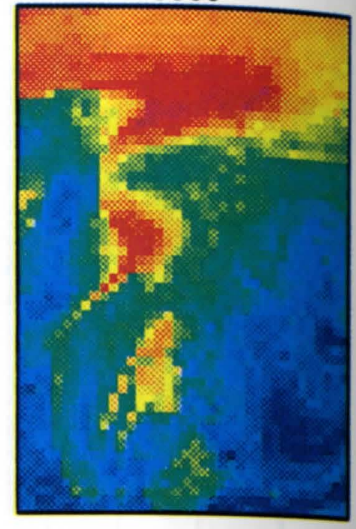

1400

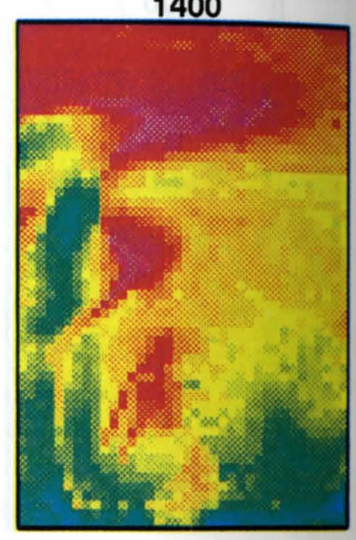

2200

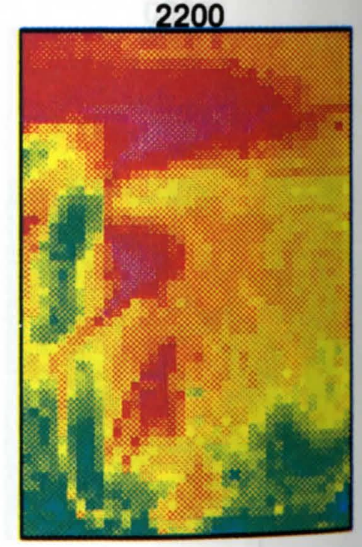

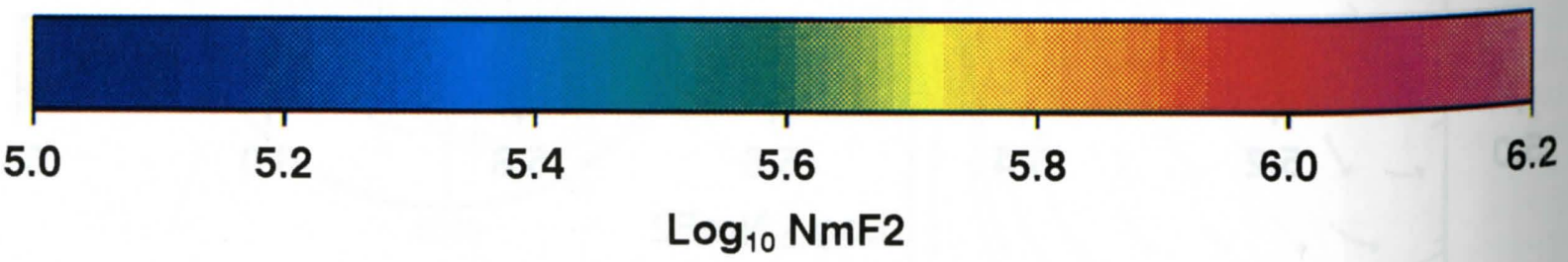

Plate 2. $N_{m} F_{2}$ in the patch simulation region over a 24-hour period for equinox and solar maximum conditions and for the same 12 UTs shown in Plate 1. The coordinate system and color key are the same as in Plate 1.

dynamic range in Plate 2 is identical to that in Plate 1 for ease of comparison. Since the same convection modulation was used for Plates 1 and 2, the general morphology of the patch structures is the same. However, the UT variation has changed significantly. At equinox there are patches present at all UTs.
This results from the fact that the TOI is a permanent feature at equinox, in contrast to winter when the TOI is absent from 0600 to 1000 UT (Plate 1). Plate 2 shows that during this period there are patches and that the contrast between them and the background density $\left(\sim 2\right.$ to $\left.3 \times 10^{5} \mathrm{~cm}^{-3}\right)$ is stronger than at 
other UTs. This implies that at equinox, patches are strongest $\sim 0800$ UT, while in winter they are strongest $\sim 2000$ UT. The peak patch density in both cases lies between $1 \times 10^{6}$ and $2 \times$ $10^{6} \mathrm{~cm}^{-3}$. At equinox the 2000 UT period has patches, but the background densities in the polar cap are very high $\left(8 \times 10^{5}\right.$ $\mathrm{cm}^{-3)}$ due to solar illumination.

The seasonal variation of the patch morphology is shown in Plate 3 as $12 N_{m} F_{2}$ simulation snapshots taken at two different UTs for each of 6 days that span the summer-equinoxwinter period. Two UTs have been selected to emphasize the winter patch modulation. These times are $0000 \mathrm{UT}$, which has patches in winter, and 0900 UT, which does not. Patches are present during all seasons, although they are absent at certain UTs in winter. Surprisingly, summer patches are present (see Plate 3 , left). In summer the patch intensity over background is only tens of percent, reaching a maximum of $\sim 50 \%$ (see Figure 5). The UT patch modulation is smallest in summer, with the 0000 and 0900 UT plots looking very similar. This difference systematically grows as the season changes from summer to equinox and then to winter.

Plate 3 also shows a clear winter anomaly in which the sunlit winter densities exceed those in summer by almost a factor of 2. This arises from both a change in the MSIS-86 atmosphere and a reduction in exospheric temperature in winter that reduces the plasma recombination rates. This latter mechanism is also, in part, responsible for the structure observed in summer (Plate 3, left panels). The low-density (blue) regions in the dusk sector of the summer polar cap are associated with the kilometer per second plasma flows (compare to Figure $3 \mathrm{~b}$ ). The enhanced density (red) regions are those dominated by upward $\mathbf{E} \times \mathbf{B}$ transport in sunlight.

From both the seasonal and UT viewpoints the patch morphology is complex. Observationally, a patch is defined by simultaneous observation of extended high $N_{m} F_{2}$ densities adjacent to regions of low density. In summer this ratio would be close to 1 , whereas in winter it could be 1 (low isotropic densities) or high, extending up to 10 . Section 5 defines a model patch-to-background $N_{m} F_{2}$ ratio and uses this ratio to present the detailed seasonal and UT patch morphology.

\section{Patch-to-Background $N_{m} F_{2}$ Ratio}

Plates 1,2 , and 3 show a particular patch distribution in the polar cap ionosphere. This distribution is dependent on the adopted time variation for the plasma convection, in this case by switching between the $\mathrm{A}$ and $\mathrm{DE}$ patterns every $30 \mathrm{~min}$ The specific structure associated with these patches would, in general, not be found. Instead, it is the gross (average) patch $N_{m} F_{2}$ over the background (nonpatch) $N_{m} F_{2}$ that would be representative of patches created by different time-changing convection pattern scenarios. In order to quantify this patchto-background density ratio, we establish the following procedure for obtaining the density ratio. This procedure is not unique, and differences in the ratios presented in this section would be obtained if the definition of a patch and the background are changed, but the overall seasonal and UT morphology would remain the same.

Figure 4 shows a gray-scaled $N_{m} F_{2}$ snapshot of a simulated ionosphere containing patches in winter at 2100 UT. The $N_{m} F_{2}$ structure takes on a variety of forms. A patch refers to regions of several pixels in which the $N_{m} F_{2}$ values are greater than the surrounding values. For the remainder of this section, attention is focused on one such patch region located at 2300 MLT and $80^{\circ}$ magnetic latitude. This patch region is $5 \times 5$ pixels, i.e., about $350 \times 350 \mathrm{~km}$ in extent. Adjacent to this region at $0100 \mathrm{MLT}$ and $80^{\circ}$ magnetic latitude is a region devoid of patches which is used as the background. The background region is $5 \times 10$ pixels, i.e., $350 \times 700 \mathrm{~km}$ in extent. These two regions are highlighted in Figure 4. Our patch-to-background density ratio is then computed as the ratio of the average $N_{m} F_{2}$ density in the patch to the average $N_{m} F_{2}$ density in the background region. Clearly, larger ratios could be obtained by using the maximum density in the patch and the average background density. Since the patch and background regions are defined in MLT, different ground locations rotate under them in a 24 -hour period. However, since the regions lie close to the magnetic pole, these ground locations all lie within the polar region. The ground station Thule, which has been used extensively as a polar cap observatory, passes under these regions. An all-sky camera viewing from Thule would readily encompass both areas.

Figure 5 shows how the modeled average patch $N_{m} F_{2}$ to modeled background $N_{m} F_{2}$ varies diurnally from summer (day 205) to winter (day 355). In each panel the patch and background regions are as defined in Figure 4 . The UT modulation seen in Figure 5 is an extension of that implied by Plates 1 and 2 for winter and equinox and Plate 3 as a function of season. If instead of the regions defined in Figure 4, others were used to define the patch or background, then the absolute ratio would be modified, but the UT and seasonal trends would remain the same. The winter period (days 325 and 355) shows a minimum patch ratio from 0800 to 1200 UT and a maximum from 2000 to 2400 UT. However, the equinox period (days 265 and 295) shows a 12-hour phase shift in the times of maximum and minimum patch ratio. Also, at equinox the minimum ratio remains well above 1.5 , while in winter it approaches 1 (no patches). In summer the patches are present, but the density ratios are very small (Figure 5, days 205 and 235).

A different perspective of this season-UT patch ratio is given in Figure 6, where the patch ratio as a function of day-ofyear is given for six selected UTs. Between 1600 and 2400 UT the patches have a peak ratio of $\sim 3.2$, and this occurs during winter solstice. However, from 0400 to 1200 UT the patches have a peak ratio just below 3 , and this occurs at equinox. During this UT interval both the winter and summer patch ratios are small. Also, in general, the summer patch ratios are small $(\leq 25 \%)$ at all UTs.

The seasonal and UT morphologies of the patch ratios shown in Figures 5 and 6 are combined into a UT-seasonal color plot of the patch ratio in Plate 4. In this format, high patch ratios are color coded red, while the low ratios are blue, as indicated in the color key. The day-of-year axis begins in summer and runs through winter back to summer. With this format the large patch ratio seasonal-UT morphology is viewed as a single entity. The most noticeable feature is the midwinter absence of patches from 0800 to 1200 UT. This corresponds to the days and UTs at which no TOI occurs in the polar cap. On either side of the winter patch ratio minimum (i.e., toward equinox) the diurnal modulation is small; indeed, patches are present at all UTs. The highest patch ratios are found over a 100-day period centered on winter solstice between 2000 and 0300 UT. As before, the Plate 4 results are for the patch and background regions defined in Figure 4. In summary, Plate 4 shows that the TOI coupled with time dependent convection does not produce significant patch ratios in the middle of winter between 0800 and 1200 UT in the northern hemisphere; other than this, equinox and winter 


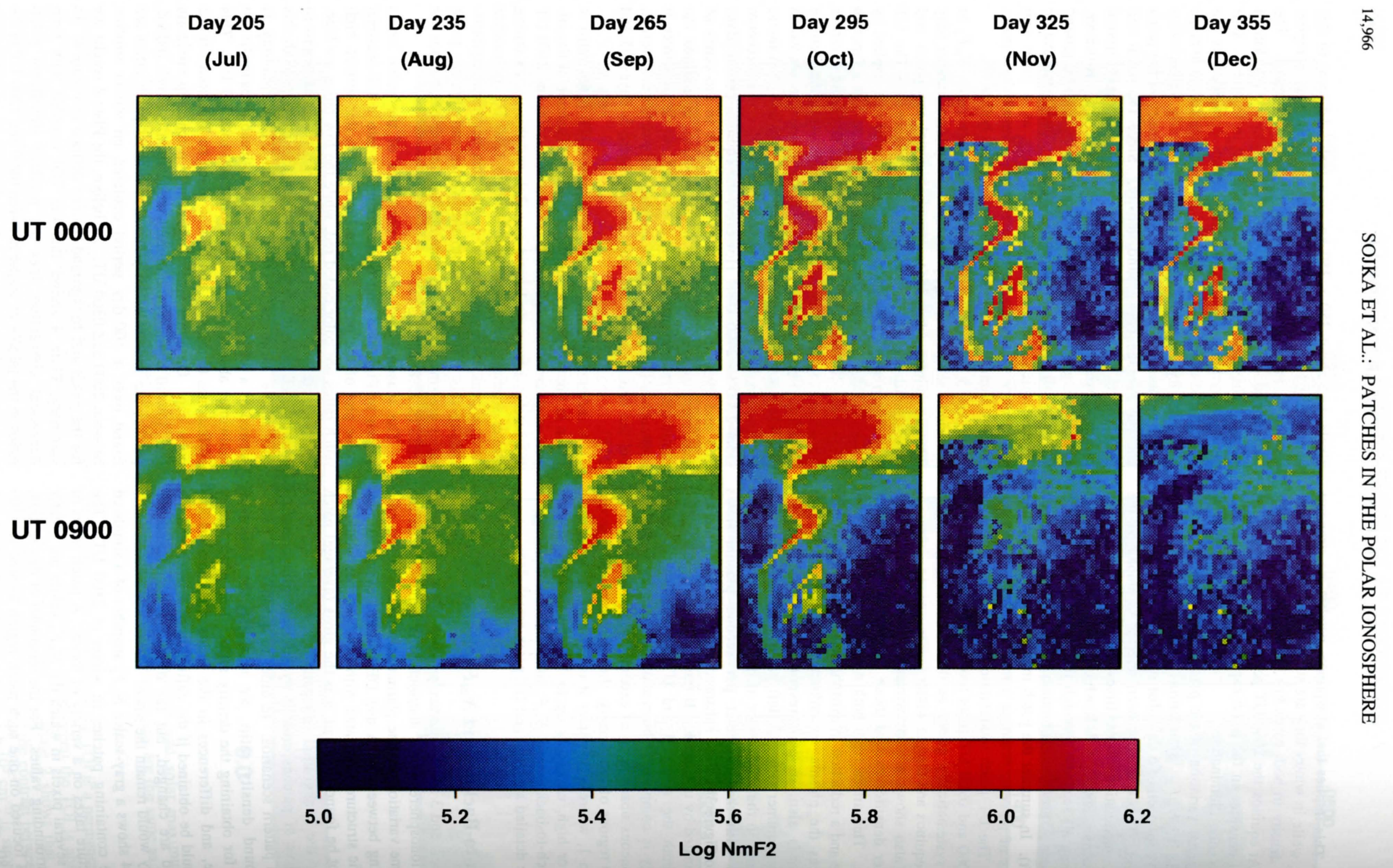

Plate 3. Seasonal variation of patches at two UTs. The patches are generated by switching between the A and DE convection patterns [Heppner and Maynard, 1987], as in Plates 1 and 2. Each panel is an $N_{m} F_{2}$ snapshot using the same color key and coordinate system as in Plates 1 and 2. 


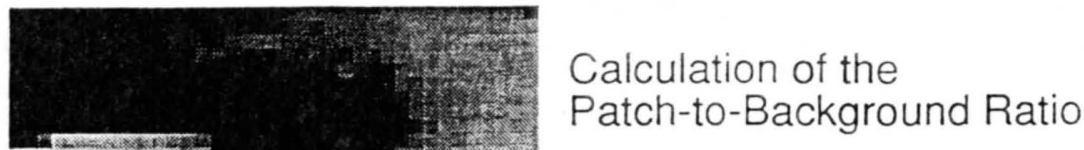

\section{Patch-to-Background Ratio}

Average the $\mathrm{NmF2}$ values inside

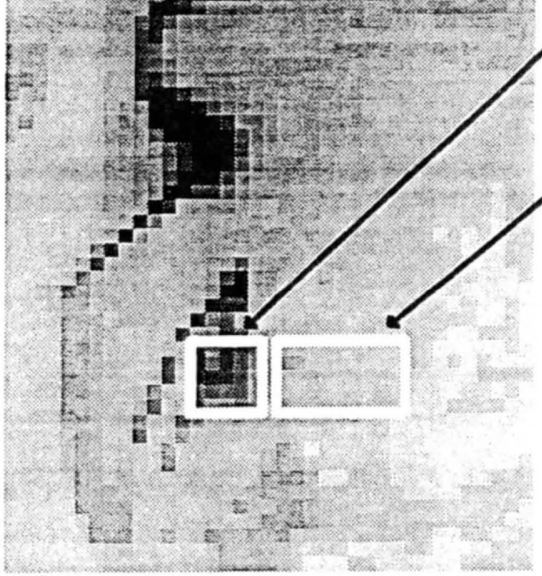

this box to obtain "Patch" NmF2

Average the $\mathrm{NmF2}$ values inside this box to obtain "Background" NmF2

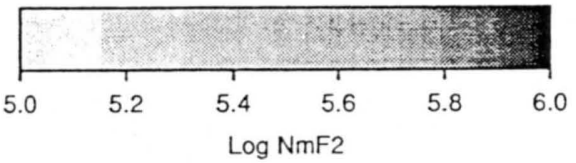

If instead of averaging, the peak patch value and the minimum background value are used, this results in an extra factor of 2 to 3 . In this case the ratio can be as high as 10 .

Figure 4. Definition of the regions used in the patch-to-background density ratio calculation.

solstice provide conditions under which large patch-tobackground density ratios can form. Even in summer, weak patching is expected.

\section{Discussion}

In this study we have simulated the formation and transport of patches. The model predicts that patch occurrence in the polar cap depends on both UT and season. The results we obtained are based upon using a specific time-varying convection electric field combined with the UT dynamics of the TOI. Sojka et al. [1993] showed that for three different time-varying convection configurations, similar patches are formed. Hence we infer that the seasonal and UT morphology of patches shown in this study is generally applicable to ionospheric polar patches under southward IMF conditions. In fact, other convection patterns have been used in our TDIM simulations, and the patches created have the same morphology as shown in this paper.

Observations of the patch UT (diurnal) effect have been made and published [Buchau et al., 1985]. These measurements were made from Thule, Greenland ( $\left.86^{\circ} \mathrm{CGL}\right)$, using a digisonde 128 PS. These ionosonde soundings have directional sensitivity as well as the ability to infer the net convection speed and direction. Hence these observations were able to identify the patches as well as verify that the convection in the polar cap was antisunward (i.e., that the IMF was southward). Buchau et al. [1985, Figure 1], described a 5 day period from December 4 to 10,1983 , that shows enhanced plasma densities between $\sim 1200$ and 2400 UT. This is also the time when the strongest patches are seen. Buchau et al. [1985] show how this diurnal variation is consistent with a solar illumination mechanism. These data are also consistent with our Plate 4 UT modulation for the appropriate December 4 to 10 period. In Plate 4, patches are predicted to disappear from 0800 to 1200 UT. When comparing with these observations, it should be noted that the ionosphere shows a much finer structuring than we can model. It is the overall morphology of the "parent" patches that we model. This UT modulation is, in fact, the same mechanism used by de la Beaujardiere et al. [1985] to explain their observations.

More interesting is the Buchau et al. [1985] January observations. Two periods of observations were carried out; January 20 to 27, 1982, and January 1983 [Buchau et al., 1983 Figures 6 and 8]. In this case the $S_{4}$ scintillation is presented; the authors have shown that the $S_{4}$ index is correlated with the degree of patches present in the polar cap. Their observations show almost no diurnal modulation of the scintillation (patches) and that during the 0600 to 1200 UT period the scintillation index is comparable with that at other times. The authors were not able to reconcile these observations with the December diurnal variations. These January observations are, however, relatively consistent with our Plate 4 prediction for the late January period (days 20 to 30 ). During this period and to day 60 our model predicts that a strong patch intensity should be present all day at a polar cap location like Thule. Indeed, days near day 60 and onward would have a maximum patch intensity at $\sim 0700 \mathrm{UT}$, rather than at the December maximum $\sim 2200$ UT.

These observations of patch $f_{0} F_{2}$ and scintillation are the most extensive data that we have found for clearly southward IMF. Extensive ionospheric studies of plasma irregularities and scintillations have been carried out, but the data have not been binned according to the IMF orientation. This latter constraint is critical for comparisons with our model. The second aspect of our study is the seasonal dependence of 
Patch - to - Background Ratio

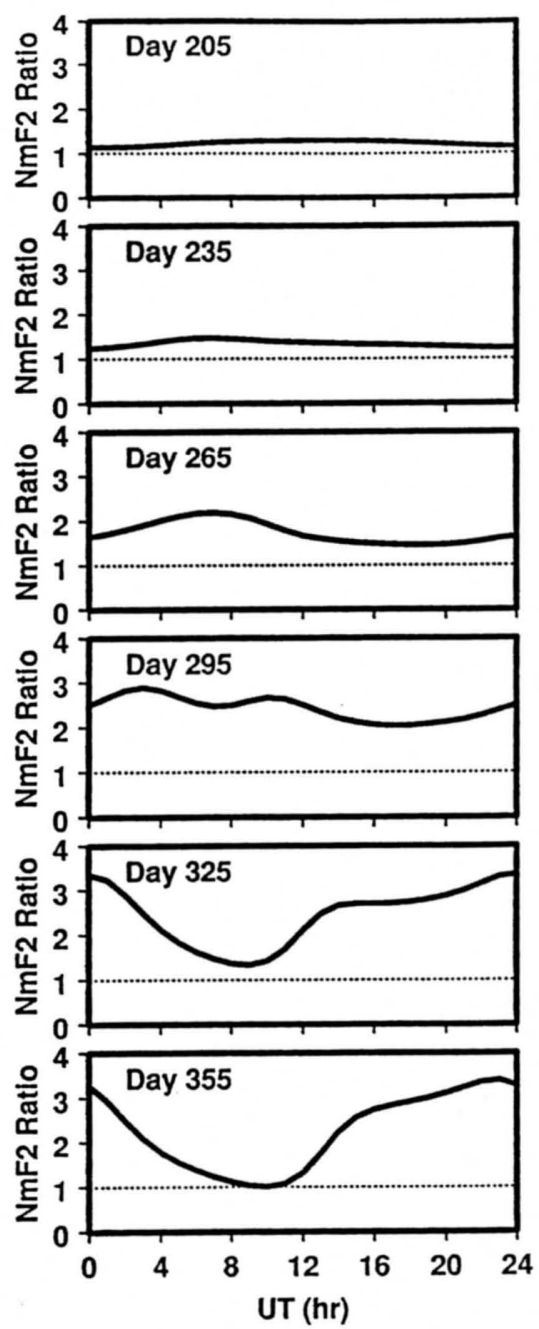

Figure 5. UT variation of the patch-to-background density ratio on 6 different days ranging from (top) summer to (bottom) winter.

patches. Observationally, patches are found most frequently and with the largest intensity in winter. However, no definitive experimental study has been done. As in our simulations, observations also show that patches, although weak, are present in summer (J. Buchau, private communication, 1992). Our discussions about the Buchau et al. [1985] December and January observations provide good evidence that the complex patch seasonal-UT dependence predicted by our model (Plate 4) is, in fact, observed.

\section{Conclusions}

This study predicts that the seasonal and UT dependencies of polar cap $F$ region patches are complex (Plate 4). (1) At winter solstice, \pm 20 days, no patches form during the 0600 1200 UT period. (2) Seasonally, the most intensive patch formation occurs at winter solstice, \pm 20 days, from 2000 to 0300 UT. (3) Between winter solstice and both equinoxes there is a period of $\sim 40$ days when strong patch formation can occur throughout the day. (4) During the summer-to-equinox periods, patches still form, but their intensity is only a few to tens of percent above that of the background ionosphere.
Patch - to - Background Ratio
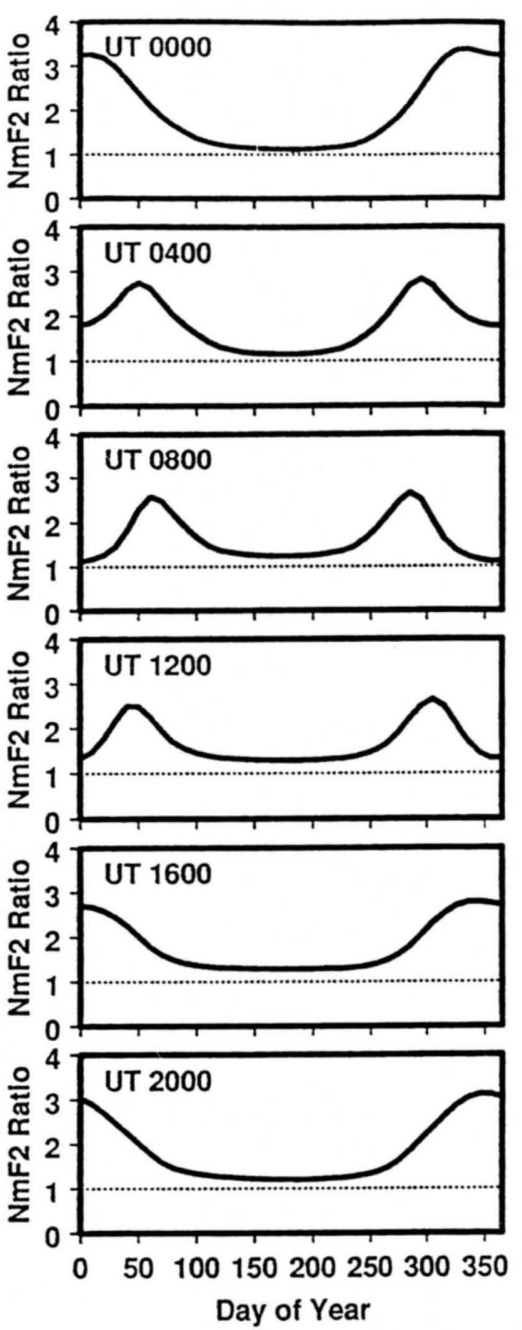

Figure 6. Seasonal variation of the patch-to-background density ratio at six different UTs.

Although our model generates patches with $100-\mathrm{km}$ to $1000-\mathrm{km}$ scale sizes, patches or irregular structures also form at smaller scale sizes. As described in the introduction, certain plasma instability mechanisms will be triggered by the patch density gradients formed by our model, while other mechanisms would contribute independently to the overall population of patches. On the basis of the Plate 4 results we can predict a method of distinguishing between some of the patch formation mechanisms. Namely, during the winter solstice 0600-1200 UT period (at a Thule type location), patches formed by our time-varying electric field mechanism would be absent (blue "hole" at winter). Hence observations made during this period would be able to deduce the extent to which other patch formation mechanisms operate. Observational campaigns, such as those under the auspices of CEDAR, are ideal for such studies. Perhaps comprehensive data for southward IMF during this period already exist. Of particular relevance would be the coordinated observation of the cleft-cusp regions. It is here that other patch mechanisms are expected to operate; these being associated with large electric fields and particle precipitation, which would reduce or enhance the plasma density. We are continuing to use the TDIM to systematically study how such alternative mechanisms would produce patches in the polar ionosphere. 

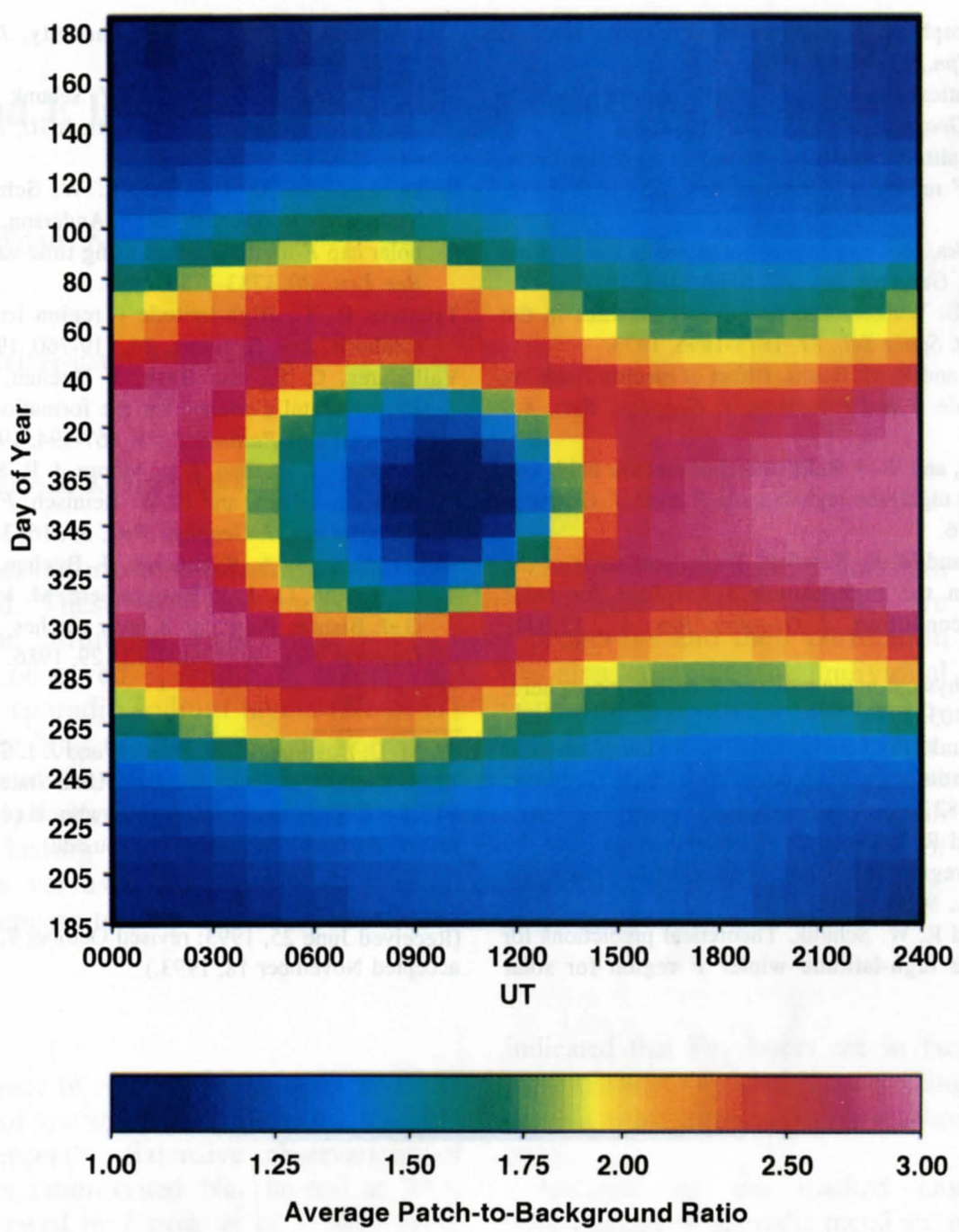

Plate 4. Patch-to-background $N_{m} F_{2}$ ratio as a function of UT and season. The ratio is computed as described in the text and illustrated in Figure 4. Ratios are represented by colors defined in the color key.

Acknowledgments. This research was supported in part by NASA grant NAG5-1484 and NSF grants ATM-89-13230, ATM-93-02165, and ATM-93-08163 to Utah State University. Helpful discussions with Dwight Decker and David Anderson are acknowledged.

The Editor thanks J. C. Foster and another referee for their assistance in evaluating this paper.

\section{References}

Anderson, D. N., J. Buchau, and R. A. Heelis, Origin of density enhancements in the winter polar cap ionosphere, Radio Sci., 23, 513-519, 1988.

Bowline, M., J. J. Sojka, and R. W. Schunk, UT control of $\mathrm{N}_{e}$ patches in the polar ionosphere (abstract), EoS Trans. AGU, 73 (43), Fall Meeting Suppl., 416, 1992.

Buchau, J., B. W. Reinisch, E. J. Weber, and J. G. Moore, Structure and dynamics of the winter polar cap $F$ region, Radio Sci., 18, 995-1010, 1983

Uuchau, J., E. J. Weber, D. N. Anderson, H. C. Carlson, Jr., J. G. Moore, B. W. Reinisch, and R. C. Livingston, Ionospheric structures in the polar cap: Their origin and relation to $250-\mathrm{MHz}$ scintillation, Radio Sci., 20, 325-338, 1985.

ecker, D. T., R. Sheehan, D. N. Anderson, and R. Heelis, Modeling polar cap $F$ layer patches, EoS Trans. AGU, 73(43), Fall Meeting Suppl., 413, 1992. de la Beaujardiere, O., V. B. Wickwar, G. Caudal, J. M. Holt, D. J. Craven, L. A. Frank, L. H. Brace, D. S. Evans, J. D. Winningham, and R. A. Heelis, Universal time dependence of nighttime $F$ region densities at high latitudes, J. Geophys. Res., 90, 4319-4332, 1985.

Foster, J. C., Storm time plasma transport at middle and high latitudes, $J$. Geophys. Res., 98, 1675-1689, 1993.

Foster, J. C., and J. R. Doupnik, Plasma convection in the vicinity of the dayside cleft, J. Geophys. Res., 89, 9107-9113, 1984.

Hardy, D. A., M. S. Gussenhoven, R. Raistrick, and W. J. McNeil, Statistical and functional representations of the pattern of auroral energy flux, number flux, and conductivity, J. Geophys. Res., 92, 12,275-12,294, 1987.

Hedin, A. E., MSIS-86 thermospheric model, J. Geophys. Res., 92, 4649-4662, 1987.

Heppner, J. P., and N. C. Maynard, Empirical high-latitude electric field models, J. Geophys. Res., 92, 4467-4489, 1987.

Knipp, D. J., et al., Ionospheric convection response to slow, strong variations in a northward interplanetary magnetic field: A case study for January 14, 1988, J. Geophys. Res., 98, 19,273-19,292, 1993. (Correction, J. Geophys. Res., 99, 6541, 1994.)

Knudsen, W. C., Magnetospheric convection and the high-latitude $\mathrm{F}_{2}$ ionosphere, J. Geophys. Res., 79, 1046-1055, 1974.

Lockwood, M., and H. C. Carlson, Jr., The production of polar cap electron density patches by transient magnetopause reconnection, Geophys. Res. Lett., 19, 1731-1734, 1992. 
Sato T., Morphology of ionospheric F2 disturbances in the polar regions, Rep. Ionos. Space Res. Jpn., 13, 91-95, 1959.

Schunk, R. W., A mathematical model of the middle and high latitude ionosphere, Pure Appl. Geophys., 127, 255-303, 1988.

Schunk, R. W., and W. J. Raitt, Atomic nitrogen and oxygen ions in the daytime high-latitude $F$ region, J. Geophys. Res., 85, 1255-1272, 1980.

Schunk, R. W., and J. J. Sojka, Ion temperature variations in the daytime high-latitude $F$ region, J. Geophys. Res., 87, 5169-5183, 1982.

Schunk, R. W., and J. C. G. Walker, Theoretical ion densities in the lower ionosphere, Planet. Space Sci., 21, 1875-1896, 1973.

Schunk, R. W., W. J. Raitt, and P. M. Banks, Effect of electric fields on the daytime high-latitude $E$ and $F$ regions, J. Geophys. Res., 80, 3121-3130, 1975.

Schunk, R. W., P. M. Banks, and W. J. Raitt, Effect of electric fields and other processes upon the nighttime high-latitude $F$ layer, J. Geophys. Res., 81, 3271-3282, 1976.

Schunk, R. W., J. J. Sojka, and M. D. Bowline, Theoretical study of the electron temperature in the high-latitude ionosphere for solar maximum and winter conditions, J. Geophys. Res., 91, 12,041$12,054,1986$.

Sojka, J. J., Global scale, physical models of the $F$ region ionosphere, Rev. Geophys., 27, 371-403, 1989.

Sojka, J. J., and R. W. Schunk, Predicted diurnal variations of electron density for three high-latitude incoherent scatter radars, Geophys. Res. Lett., 9, 143-146, 1982.

Sojka, J. J., W. J. Raitt, and R. W. Schunk, A theoretical study of the high-latitude winter $F$ region at solar minimum for low magnetic activity, J. Geophys. Res., 86, 609-621, 1981a.

Sojka, J. J., W. J. Raitt, and R. W. Schunk, Theoretical predictions for ion composition in the high-latitude winter $F$ region for solar minimum and low magnetic activity, J. Geophys. Res., 86, 2206. 2216, 1981b.

Sojka, J. J., M. Bowline, and R. W. Schunk, Simulation of $\mathrm{N}_{e}$ patches in the polar ionosphere, EoS Trans. AGU, 73(43), Fall Meeting Suppl 414, 1992.

Sojka, J. J., M. D. Bowline, R. W. Schunk, D. T. Decker, C. E Valladares, R. Sheehan, D. N. Anderson, and R. A. Heelis, Modeling polar cap $F$-region patches using time varying convection, Geophys. Res. Lett., 20, 1783-1786, 1993.

Tsunoda, R. T., High-latitude F region irregularities: A review and synthesis, Rev. Geophys., 26, 719-760, 1988.

Valladares, C. E., Su. Basu, J. Buchau, and E. Friis-Christensen, Experimental evidence for the formation and entry of patches into the polar cap, Radio Sci., 29, 167-194, 1994.

Weber, E. J., J. Buchau, J. G. Moore, J. R. Sharber, R. C. Livingston, J. D. Winningham, and B. W. Reinisch, $F$ layer ionization patches in the polar cap, J. Geophys. Res., 89, 1683-1694, 1984.

Weber, E. J., J. A. Klobuchar, J. Buchau, H. C. Carlson, Jr., R. C. Livingston, O. de la Beaujardiere, M. McCreday, J. G. Moore, and G. J. Bishop, Polar cap $F$ layer patches: Structure and dynamics, J. Geophys. Res., 91, 12,121-12,129, 1986.

M. D. Bowline, R. W. Schunk, and J. J. Sojka, Center for Atmospheric and Space Sciences, Utah State University, Logan, UT 84322-4405. (e-mail: Internet. bowline@cc.usu.edu; schunk@cc.usu.edu.; sojka@cc.usu.edu)

(Received June 25, 1993; revised October 7, 1993; accepted November 18, 1993.) 